\title{
Control of Robotic Mobility-On-Demand Systems: a Queueing-Theoretical Perspective
}

\author{
Rick Zhang and Marco Pavone \\ Department of Aeronautics and Astronautics, Stanford University \\ \{rickz, pavone\}estanford.edu
}

\begin{abstract}
In this paper we present and analyze a queueingtheoretical model for autonomous mobility-on-demand (MOD) systems where robotic, self-driving vehicles transport customers within an urban environment and rebalance themselves to ensure acceptable quality of service throughout the entire network. We cast an autonomous MOD system within a closed Jackson network model with passenger loss. It is shown that an optimal rebalancing algorithm minimizing the number of (autonomously) rebalancing vehicles and keeping vehicles availabilities balanced throughout the network can be found by solving a linear program. The theoretical insights are used to design a robust, real-time rebalancing algorithm, which is applied to a case study of New York City. The case study shows that the current taxi demand in Manhattan can be met with about 8,000 robotic vehicles (roughly $70 \%$ of the size of the current taxi fleet operating in Manhattan). Finally, we extend our queueingtheoretical setup to include congestion effects, and we study the impact of autonomously rebalancing vehicles on overall congestion. Collectively, this paper provides a rigorous approach to the problem of system-wide coordination of autonomously driving vehicles, and provides one of the first characterizations of the sustainability benefits of robotic transportation networks.
\end{abstract}

\section{INTRODUCTION}

According to United Nations estimates, urban population will double in the next 30 years [28]. Given the limited availability for additional roads and parking spaces in current (mega)-cities, private automobiles appear as an unsustainable solution for the future of personal urban mobility [20]. Arguably, one of the most promising approaches to cope with this problem is one-way vehicle sharing with electric cars (referred to as Mobility-On-Demand, or MOD), which directly targets the problems of parking spaces, pollution, and low vehicle utilization rates [20]. Limited-size MOD systems with human-driven vehicles have recently been deployed in several European and American cities [6]. However, such systems lead to vehicle imbalances, that is some stations become rapidly depleted of vehicles while others have too many, due to some stations being more popular than others. Somewhat surprisingly, even if the transportation network is symmetric (that is, the underlying network topology is a regular grid and arrival rates and routing choices of customers at all nodes are uniform), the stochastic nature of customer arrivals to the stations will quickly drive the system out of balance and hence to instability (since the customer queue will grow without bound at some stations) [10].

The related problem of rebalancing in the increasingly popular bike-sharing systems is solved using trucks which can carry many bikes at the same time, and algorithms have very recently been developed to optimize the truck routes [7, 8]. Since this approach is not feasible with cars, the work in [25] considers the possibility of hiring a team of rebalancing drivers whose job is to rebalance the vehicles throughout the transportation network. However, with this approach the rebalancing drivers themselves become unbalanced, and one needs to "rebalance the rebalancers," which significantly increases congestion and costs [25]. Another option would be to incentivize ride sharing [1], which, unfortunately, defeats the purpose of a MOD system to ensure personal mobility.

Recently, a transformational technology has been proposed in [22, 5], whereby driverless electric cars shared by the customers provide on-demand mobility. Autonomous driving holds great promise for MOD systems because robotic vehicles can rebalance themselves (thus eliminating the rebalancing problem at its core), enable system-wide coordination, free passengers from the task of driving, and potentially increase safety. Indeed, robotic vehicles specifically designed for personal urban mobility are already being tested (e.g., the Induct Navia vehicle [14], the General Motors' EN-V vehicle [12], and the Google car [9]). Yet, little is known about how to design and operate robotic transportation networks [22].

Statement of contributions: The objective of this paper is to develop a model of, study rebalancing algorithms for, and evaluate the potential benefits of a MOD system where mobility is provided by driverless cars (henceforth referred to as autonomous MOD system). Rebalancing algorithms for autonomous MOD systems have been investigated in [22] under a fluidic approximation (i.e., customers and vehicles are modeled as a continuum). While this approach provides valuable insights for the operation of an autonomous MOD system, by its very nature, it does not provide information about the effect of stochastic fluctuations in the system (e.g., due to the customers' arrival process) and, most importantly, it does not allow the computation of key performance metrics such as availability of vehicles at stations and customer waiting times. This motivates the queueing-theoretical approach considered in this paper. In this respect, our work is related to [11, 29], where a transportation network comprising traditional (i.e., human-driven) shared vehicles is modeled within the framework of Jackson networks [24]. The key technical difference is that in this paper we address the problem of synthesizing a rebalancing policy, rather than analyzing the evolution of the vehicle distribution under the customers' routing choices. Our work is also related to the Dynamic Vehicle Routing problem [4, 21] and, more specifically, to the Dynamic Pickup and Delivery Problem (DPDP) [2, 27]. However, current DPDP works do not explicitly consider rebalancing, and system performance results, whenever available, are usually limited to asymptotic cases where the load on the system approaches 0 or approaches the capacity of the system [27].

Specifically, the contribution of this paper is fourfold. First, we propose a queueing-theoretical model of an autonomous MOD system cast within a Jackson network model (valid 
under any load condition). Second, we study the problem of synthesizing rebalancing algorithms, where the control objective is to minimize the number of (autonomously) rebalancing vehicles on the roads while keeping vehicle availabilities balanced throughout the network. Remarkably, we show that under certain assumptions an optimal policy can be solved as a linear program. Third, we apply our theoretical results to a case study of New York City, which shows that the current taxi demand in Manhattan can be met with about 8,000 robotic vehicles (roughly $70 \%$ of the size of the current taxi fleet operating in Manhattan). This shows the potential of autonomous MOD systems. Finally, by leveraging our queueingtheoretical setup, we study the potential detrimental effect of rebalancing on traffic congestion (rebalancing vehicles, in fact, increase the number of vehicles on the roads). Our study suggests that while autonomously rebalancing vehicles can have a detrimental impact on traffic congestion in alreadycongested systems, in most cases this is not generally a concern as rebalancing vehicles "tend" to travel along less congested roads. To the best of our knowledge, this is the first paper to provide a rigorous, stochastic approach to the problem of system-wide coordination of autonomously driving vehicles.

Organization: The remainder of the paper is structured as follows: In Section [I] we briefly review some well-known results of queueing networks, specifically Jackson networks. In Section [II] we show how to model an autonomous MOD system with rebalancing within a Jackson network model. In Section $[\mathrm{IV}]$ we formulate the optimal rebalancing problem, we show that it can be solved via a linear program, we provide an iterative algorithm to compute relevant performance metrics (chiefly, vehicle availability at stations), and we use the theoretical insights to design a robust, real-time rebalancing policy. In Section $\mathrm{V}$ we apply our model and algorithms to a case study of New York City, while in Section VI we extend our queueing-theoretical setup to include congestion effects. Finally, in Section VII we draw our conclusions, and present directions for future research.

\section{BACKGROUND MATERIAL}

In this section we review some key results from the theory of Jackson networks, on which we will rely extensively later in the paper. Consider a network consisting of $|\mathcal{N}|$ first-come first-serve nodes, or queues, where $\mathcal{N}$ represents the set of nodes in the network. Discrete customers arrive from outside the network according to a stochastic process or move among the nodes. Customers that arrive at each node are serviced by the node, and proceed to another node or leave the system. A network is called closed if the number of customers in the system remains constant and no customers enter or leave the network. A Jackson network is a Markov process where customers move from node to node according to a stationary routing distribution $r_{i j}$ and the service rate $\mu_{i}(n)$ at each node $i$ depends only on the number of customers at that node, $n$ [24, p.9]. For the remainder of this paper, we consider only closed networks. The state space of a closed Jackson network with $m$ customers is given by $\Omega_{m}=\left\{x=\left(x_{1}, x_{2}, \ldots, x_{|\mathcal{N}|}\right): \sum_{i=1}^{|\mathcal{N}|} x_{i}=m, x_{i} \in \mathbb{Z}_{\geq 0}\right\}$, where $x_{i}$ is the number of customers at node $i$. Jackson networks are known to admit a product-form stationary distribution, where the stationary distribution of the network is given by a product of the distribution of each node. In equilibrium, the throughput at each node (average number of customers moving through a node per unit time) satisfies the traffic equations

$$
\pi_{i}=\sum_{j \in \mathcal{N}} \pi_{j} r_{j i} \quad \forall i \in \mathcal{N} .
$$

For a closed network, equation (1) does not have a unique solution, and $\pi=\left(\begin{array}{llll}\pi_{1} & \pi_{2} & \ldots & \pi_{N}\end{array}\right)^{T}$ only determines the throughput up to a constant factor, and is therefore called the relative throughput. The stationary distribution of the network is given by

$$
\mathbb{P}\left(x_{1}, x_{2}, \ldots, x_{|\mathcal{N}|}\right)=\frac{1}{G(m)} \prod_{j=1}^{|\mathcal{N}|} \pi_{j}^{x_{j}} \prod_{n=1}^{x_{j}} \mu_{j}(n)^{-1} .
$$

The quantity $G(m)$ is the normalization constant needed to make $\mathbb{P}\left(x_{1}, x_{2}, \ldots, x_{|\mathcal{N}|}\right)$ a probability measure, and is given by $G(m)=\sum_{x \in \Omega_{m}} \prod_{j=1}^{|\mathcal{N}|} \pi_{j}^{x_{j}} \prod_{n=1}^{x_{j}} \mu_{j}(n)^{-1}$. Many performance measures of closed Jackson networks can be expressed in terms of the normalization factor $G(m)$. In [24, p.27], it is shown that the actual throughput of each node is given by

$$
\Lambda_{i}(m)=\pi_{i} G(m-1) / G(m) .
$$

One can further define the quantity

$$
\gamma_{i}=\pi_{i} / \mu_{i}(1) \quad \forall i \in \mathcal{N},
$$

where $\gamma_{i}$ is referred to as the relative utilization of node $i$. Lavenberg [16, p.128] showed that the marginal distribution of the queue length variable $X_{i}$ at node $i \in \mathcal{N}$ is given by

$$
\mathbb{P}\left(X_{i}=x_{i}\right)=\gamma_{i}^{x_{i}}\left[G\left(m-x_{i}\right)-\gamma_{i} G\left(m-x_{i}-1\right)\right] / G(m) .
$$

A quantity of interest is the probability that a node has at least 1 customer, which we refer to as the availability of node $i, A_{i}(m)$. This is given by

$$
\begin{aligned}
A_{i}(m) & =1-P\left(X_{i}=0\right) \\
& =1-\frac{G(m)-\gamma_{i} G(m-1)}{G(m)}=\frac{\gamma_{i} G(m-1)}{G(m)} .
\end{aligned}
$$

\section{Model Description and Problem Formulation}

\section{A. Model of autonomous MOD system}

In this paper, we model an autonomous MOD system within a queueing theoretical framework. Consider $N$ stations placed within a given geographical area and $m$ (autonomous) vehicles that provide service to passengers. Within our queueing framework, the vehicles correspond to "customers" described in Section [II. Henceforth, the term customers will refer to passengers rather than to vehicles. Customers arrive at each station $i$ according to a time-invariant Poisson process with rate $\lambda_{i} \in \mathbb{R}_{>0}$. Upon arrival, a customer at station $i$ selects a destination $j$ with probability $p_{i j}$, where $p_{i j} \in \mathbb{R}_{\geq 0}, p_{i i}=0$, and $\sum_{j} p_{i j}=1$. Furthermore, we assume that the probabilities $\left\{p_{i j}\right\}_{i j}$ constitute an irreducible Markov chain. If there are vehicles parked at station $i$, the customer takes the vehicle and travels to her/his selected destination. Instead, if the station is empty of vehicles, the customer immediately leaves the system. This type of customer model will be referred to as a "passenger loss" model (as opposed to a model where passengers form a queue at each station). A consequence of 
the passenger loss model is that the number of customers at each station at a fixed instant in time is 0 (since customers either depart immediately with a vehicle or leave the system). We assume that each station has sufficiently many parking spaces so that vehicles can always immediately park upon arrival at a station. The travel time from station $i$ to station $j$ is an exponentially distributed random variable with mean equal to $T_{i j} \in \mathbb{R}_{>0}$. The travel times for the different passengers are assumed to constitute an independently and identically distributed sequence (i.i.d.). The vehicles can autonomously travel throughout the network in order to rebalance themselves and best anticipate future demand. The performance criterion that we are interested in is the availability of vehicles at each station (the probability that at least one vehicle is at the station or conversely the probability that a customer will be lost).

A few comments are in order. First, our model captures well the setup with impatient customers, not willing to make use of a MOD system if waiting is required. In this respect, our model appears to be suitable for studying the benefits of autonomous MOD systems whenever high quality of service (as measured in terms of average waiting times for available vehicles) is required. From a practical standpoint, the loss model assumption significantly simplifies the problem, as it essentially allows us to decouple the "vehicle process" and the "customer process" (see Section III-B). Second, travel times, in practice, do not follow an exponential distribution. However we make this assumption as (i) it simplifies the problem considerably, and (ii) reasonable deviations from this assumption have been found not to alter in any practical way the predictive accuracy of similar spatial queueing models used for vehicle routing [15]. Third, the assumption that the probabilities $\left\{p_{i j}\right\}_{i j}$ constitute an irreducible Markov chain appear appropriate for dense urban environments. Finally, our model does not consider congestion, which is clearly a critical aspect for the efficient system-wide coordination of autonomous vehicles in a MOD system. The inclusion of congestion effects will be discussed in Section VI

\section{B. Casting an autonomous MOD system into a Jackson model}

The key idea to cast an autonomous MOD system into a Jackson model is to consider an abstract queueing network where we identify the stations with single-server (SS) nodes (also referred to as "station" nodes) and the roads with infiniteserver (IS) nodes (also referred to as "road" nodes). Assume, first, the simplified scenario where vehicles do not perform rebalancing trips (in which case, the model is essentially identical to the one in [11]). In this case, at each station node, vehicles form a queue while waiting for customers and are "serviced" when a customer arrives. A vehicle departing from a SS node moves to the IS node that connects the origin to the destination selected by the customer. After spending an exponentially distributed amount of time in the IS node (i.e., the "travel time"), the vehicle moves to the destination SS node. According to our model, once a vehicle leaves a SS (station) node $i$, the probability that it moves to the IS (road) node $i j$ is $p_{i j}$. The vehicle then moves to SS (station) node $j$ with probability 1 . Note that with this identification we have modeled a MOD system (at least in the case without rebalancing) as a closed queueing network with respect to the vehicles. Note that the road queues are modeled as infiniteserver queues as the model does not consider congestion effects (in Section VI we will see that if congestion is taken into account the road queues become finite-server queues).

More formally, denote by $S$ the set of single-server nodes and $I$ the set of infinite-server nodes. Each station is mapped into a SS node, while each road is mapped into an IS node. The set of all nodes in the abstract queueing network is then given by $\mathcal{N}=S \cup I$. Since each SS node is connected to every other SS node, and since $p_{i i}=0$ (hence the road node $i i$ does not need to be represented), the number of nodes in the network is given by $N(N-1)+N=N^{2}$, in other words, $|\mathcal{N}|=N^{2}$. For each IS node $i \in I$, let $\operatorname{Parent}(i)$ and $\operatorname{Child}(i)$ be the origin and destination nodes of $i$, respectively. As explained before, vehicles in the abstract queueing network move between SS nodes and IS nodes according to the routing matrix $\left\{r_{i j}\right\}_{i j}$ :

$$
r_{i j}= \begin{cases}p_{i l} & i \in S, j \in I \text { where } i=\operatorname{Parent}(j), l=\operatorname{Child}(j), \\ 1 & i \in I, j \in S \text { where } j=\operatorname{Child}(i), \\ 0 & \text { otherwise, }\end{cases}
$$

where the first case corresponds to a move from a SS node to an IS node (according to the destination selected by a customer), and the second case to a move from an IS node to the unique SS node corresponding to its destination. Furthermore, the service times at each node $i \in \mathcal{N}$ are exponentially distributed with service rates given by

$$
\mu_{i}(n)= \begin{cases}\lambda_{i} & \text { if } i \in S, \\ n \cdot \mu_{j k} & \text { if } i \in I, j=\operatorname{Parent}(i), k=\operatorname{Child}(i),\end{cases}
$$

where $n \in\{0,1, \ldots m\}$ is the number of vehicles at node $i$, and $\mu_{j k}=1 / T_{j k}$. The first case is the case where vehicles wait for customers at stations, while the second case is the case where vehicles spend an exponentially distributed travel time to move between stations (note that the IS nodes correspond to infinite-server queues, hence the service rate is proportional to the number of vehicles in the queue). As defined, the abstract queueing network is a closed Jackson network, and hence can be analyzed with the tools discussed in Section III.

Assume, now, that we allow the vehicles to autonomously rebalance throughout the network. To include rebalancing while staying within the Jackson network framework, we focus on a particular class of stochastic rebalancing policies described as follows. Each station $i$ generates "virtual passengers" according to a Poisson process with rate $\psi_{i}$, independent of the real passenger arrival process, and routes these virtual passengers to station $j$ with probability $\alpha_{i j}$ (with $\sum_{j} \alpha_{i j}=1$ and $\alpha_{i i}=0$ ). As with real passengers, the virtual passengers are lost if the station is empty upon generation. Such class of rebalancing policies encourages rebalancing but does not enforce a rebalancing rate, which allows us to maintain tractability in the model.

One can then combine the real passenger arrival process with the virtual passenger process (assumed independent) using the independence assumption to form a model of the same form as the one described earlier in this section while taking into account vehicle rebalancing. Specifically, we consider the same set of SS nodes and IS nodes (since the transportation network is still the same). Let $\left\{A_{t}^{(i)}, t \geq 0\right\}$ be the total arrival 
process of real and virtual passengers at station $i \in S$, and denote its rate with $\tilde{\lambda}_{i}$. The process $A_{t}^{(i)}$ is Poisson since it is the superposition of two independent Poisson processes. Hence, the rate $\tilde{\lambda}_{i}$ is given by

$$
\tilde{\lambda}_{i}=\lambda_{i}+\psi_{i} .
$$

Equivalently, one can view the passenger arrival process and the rebalancing process as the result of Bernoulli splitting on $A_{t}^{(i)}$ with a probability $p_{i}$ satisfying

$$
\psi_{i}=p_{i} \tilde{\lambda}_{i}, \quad \lambda_{i}=\left(1-p_{i}\right) \tilde{\lambda}_{i} .
$$

Let us refer to passengers arriving according the the processes $\left\{A_{t}^{(i)}, t \geq 0\right\}$ as generalized passengers. The probability $\tilde{p}_{i j}$ that a generalized passenger arriving at station $i$ selects a destination $j$ is given by

$$
\begin{aligned}
\tilde{p}_{i j} & =\mathbb{P}(i \rightarrow j \mid \text { virtual }) p_{i}+\mathbb{P}(i \rightarrow j \mid \neg \text { virtual })\left(1-p_{i}\right) \\
& =\alpha_{i j} p_{i}+p_{i j}\left(1-p_{i}\right),
\end{aligned}
$$

where $\mathbb{P}(i \rightarrow j \mid$ virtual $)$ is the probability that a virtual passenger selects station $j$ as its destination, and $\mathbb{P}(i \rightarrow$ $j \mid \neg$ virtual) is the probability that a real passenger selects station $j$ as its destination. One can then identify an autonomous MOD system with rebalancing (for the specific class of rebalancing policies discussed above) with an abstract queueing network with routing matrix and service rates given, respectively, by equations (5) and (6), where $p_{i l}$ is replaced by $\tilde{p}_{i l}, \lambda_{i}$ is replaced by $\lambda_{i}$, and $r_{i j}$ is replaced by $\tilde{r}_{i j}$. In this way, the model is still a closed Jackson network model. For notational convenience, we order $\gamma_{i}$ and $\pi_{i}$ (as defined in Section IIT in such a way that the first $N$ components correspond to the $N$ stations (for example, $\gamma_{i}$ corresponds to station $i$, or the $i$ th SS node, where $i=1,2, \ldots N$ ).

As already mentioned, in order to identify an autonomous MOD system with rebalancing with a Jackson queueing model, we restricted the class of rebalancing policies to open-loop, "rebalancing promoting" policies. We will consider closedloop policies in Section IV-C

\section{Problem formulation}

Within our model, the optimization variables are the rebalancing rates $\psi_{i}$ and $\alpha_{i j}$ of the rebalancing promoting policies. One might wonder in the first place if and when rebalancing is even required. Indeed, one can easily obtain that, for the case without rebalancing [11], $\lim _{m \rightarrow \infty} A_{i}(m)=\gamma_{i} / \gamma_{S}^{\max }$, for all $i \in S$, where $\gamma_{i}$ is the relative utilization of node $i \in S, A_{i}(m)$ is the availability of vehicles at node $i \in S$ (see Section III) and $\gamma_{S}^{\max }:=\max _{i \in S} \gamma_{i}$. Hence, as $m$ approaches infinity, the set of stations $B:=\left\{i \in S: \gamma_{i}=\gamma_{S}^{\max }\right\}$ can have availability arbitrarily close to 1 while all other stations have availability strictly less than 1 regardless of $m$. In other words, without rebalancing, a MOD system will always experience passenger losses no matter how many vehicles are employed!

The above discussion motivates the need for rebalancing. The tenet of our approach is to ensure, through rebalancing, that the network is (on average) in balance, i.e., $A_{i}(m)=$ $A_{j}(m)$ for all $i, j \in S$ (or, equivalently, $\gamma_{i}=\gamma_{j}$ for all $i, j \in$ $S$, as implied by equation (4)). The motivation behind this philosophy is twofold (i) it provides a natural notion of service fairness, and (ii) it fulfills the intuitive condition that as $m$ goes to $+\infty$ the availability of each station goes to one (since in this case $\gamma_{i}=\gamma_{S}^{\max }$ for all $i$ in $S$ ). The objective then is to manipulate the rebalancing rates $\alpha_{i j}$ and $\psi_{i}$ such that all the $\gamma_{i}$ 's in $S$ are equal while minimizing the number of rebalancing vehicles on the road. Note that the average number of rebalancing vehicles travelling between station nodes $i$ and $j$ is given by $T_{i j} \alpha_{i j} \psi_{i}$. The rebalancing problem we wish to solve is then as follows:

Optimal Rebalancing Problem (ORP): Given an autonomous MOD system modeled as a closed Jackson network, solve

$$
\begin{array}{ll}
\underset{\psi_{i}, \alpha_{i j}}{\operatorname{minimize}} & \sum_{i, j} T_{i j} \alpha_{i j} \psi_{i} \\
\text { subject to } & \gamma_{i}=\gamma_{j} \\
& \sum_{j} \alpha_{i j}=1 \\
& \alpha_{i j} \geq 0, \psi_{i} \geq 0 \quad i, j \in\{1, \ldots, N\} \\
\text { where } \gamma_{i}= & \frac{\pi_{i}}{\lambda_{i}+\psi_{i}} \text { and } \pi_{i} \text { satisfies equation (1). }
\end{array}
$$

Note that to solve the ORP one would need to explicitly compute the relative throughputs $\pi$ 's using the traffic equation (1). This involves finding the 1-dimensional null space of a $\mathbb{R}^{N^{2} \times N^{2}}$ matrix, which becomes computationally expensive as the number of stations become large. Furthermore, the objective function and the constraints $\gamma_{i}=\gamma_{j}$ are nonlinear in the optimization variables. In the next section we show how to reduce the dimension of the problem to $\mathbb{R}^{N}$ and how the ORP can be readily solved as a minimum cost flow problem.

\section{Optimal ReBALANCING}

\section{A. Optimal rebalancing}

In this section, we show how the ORP can be readily solved as a minimum cost flow problem. To this purpose, we first present two key lemmas, whose proofs are given in the appendix of [30]. The first lemma shows how the traffic equations (1) can be written only in terms of the SS nodes.

Lemma IV.1 (Folding of traffic equations). Consider an autonomous MOD system modeled as a closed Jackson network as described in Section III-B. Then the relative throughputs $\pi$ 's for the SS nodes can be found by solving the reduced traffic equations

$$
\pi_{i}=\sum_{k \in S} \pi_{k} \tilde{p}_{k i} \quad \forall i \in S,
$$

where SS nodes are considered in isolation. The $\pi$ 's for the IS nodes are then given by

$$
\pi_{i}=\pi_{\text {Parent }(i)} \tilde{p}_{\text {Parent }(i) \operatorname{Child}(i)} \quad \forall i \in I .
$$

Lemma IV.2. For any rebalancing policy $\left\{\psi_{i}\right\}_{i}$ and $\left\{\alpha_{i j}\right\}_{i j}$, it holds for all $i \in S$

$$
\begin{aligned}
& \text { 1) } \gamma_{i}>0 \text {, } \\
& \text { 2) }\left(\lambda_{i}+\psi_{i}\right) \gamma_{i}=\sum_{j \in S} \gamma_{j}\left(\alpha_{j i} \psi_{j}+p_{j i} \lambda_{j}\right) \text {. }
\end{aligned}
$$

The next theorem (which represents the main result of this section) shows that we can always solve problem ORP by solving a low dimensional linear optimization problem. 
Theorem IV.3 (Solution to problem ORP). Consider the linear optimization problem

$$
\begin{array}{ll}
\underset{\beta_{i j}}{\operatorname{minimize}} & \sum_{i, j} T_{i j} \beta_{i j} \\
\text { subject to } & \sum_{\substack{j \neq i \\
j \neq 0}}\left(\beta_{i j}-\beta_{j i}\right)=-\lambda_{i}+\sum_{j \neq i} p_{j i} \lambda_{j}
\end{array}
$$

The optimization problem (13) is always feasible. Let $\left\{\beta_{i j}^{*}\right\}_{i j}$ denote an optimal solution. By setting $\psi_{i}=\sum_{j \neq i} \beta_{i j}^{*}$, $\alpha_{i i}=$ 0 , and, for $j \neq i$,

$$
\alpha_{i j}= \begin{cases}\beta_{i j}^{*} / \psi_{i} & \text { if } \psi_{i}>0 \\ 1 /(N-1) & \text { otherwise },\end{cases}
$$

one obtains an optimal solution to problem ORP.

Proof: First, we note that problem (13) is an uncapacitated minimum cost flow problem and thus is always feasible. Consider an optimal solution to problem (13), $\left\{\beta_{i j}^{*}\right\}_{i j}$, and set $\left\{\psi_{i}\right\}_{i}$ and $\left\{\alpha_{i j}\right\}_{i j}$ as in the statement of the theorem. We want to show that, with this choice, $\left\{\psi_{i}\right\}_{i}$ and $\left\{\alpha_{i j}\right\}_{i j}$ represent an optimal solution to the ORP. Since $\left\{\beta_{i j}^{*}\right\}_{i j}$ is an optimal solution to problem (13), then one easily concludes that $\left\{\psi_{i}\right\}_{i}$ and $\left\{\alpha_{i j}\right\}_{i j}$ are an optimal solution to problem

$$
\begin{array}{cl}
\underset{\psi_{i}, \alpha_{i j}}{\operatorname{minimize}} & \sum_{i, j} T_{i j} \alpha_{i j} \psi_{i} \\
\text { subject to } & \lambda_{i}+\psi_{i}=\sum_{j} \alpha_{j i} \psi_{j}+p_{j i} \lambda_{j} \\
& \sum_{j} \alpha_{i j}=1 \\
& \alpha_{i j} \geq 0, \quad \psi_{i} \geq 0
\end{array}
$$

The objective is now to show that the constraint

$$
\lambda_{i}+\psi_{i}=\sum_{j} \alpha_{j i} \psi_{j}+p_{j i} \lambda_{j}
$$

is equivalent to the constraint

$$
\gamma_{i}=\gamma_{j}
$$

Consider, first, the case where the $\left\{\alpha_{i j}\right\}_{i j}$ and $\left\{\psi_{i}\right\}_{i}$ satisfy constraint (15). Then, considering Lemma IV.2, one can write, for all $i$,

$$
\left(\sum_{j} \alpha_{j i} \psi_{j}+p_{j i} \lambda_{j}\right) \gamma_{i}=\sum_{j \in S} \gamma_{j}\left(\alpha_{j i} \psi_{j}+p_{j i} \lambda_{j}\right)
$$

Let $\varphi_{i j}:=\alpha_{j i} \psi_{j}+p_{j i} \lambda_{j}$ and $\zeta_{i j}:=\varphi_{i j} / \sum_{j} \varphi_{i j}$. (Note that $\sum_{j} \varphi_{i j}=\lambda_{i}+\psi_{i}>0$ as $\lambda_{i}>0$ by assumption.) Since $\alpha_{i i}=0$ and $p_{i i}=0$, one has $\zeta_{i i}=0$. The variables $\left\{\zeta_{i j}\right\}_{i j}$ 's can be considered as transition probabilities of an irreducible Markov chain (since, by assumption, the probabilities $\left\{p_{i j}\right\}_{i j}$ constitute an irreducible Markov chain). Then, one can rewrite equation (17) as $\gamma_{i}=\sum_{j} \gamma_{j} \zeta_{i j}$, which can be rewritten in compact form as $Z \gamma=\gamma$, where $\gamma=\left(\gamma_{1}, \ldots, \gamma_{N}\right)^{T}$ and $Z$ is an irreducible, row stochastic matrix whose $i$ th row is given by $\left[\zeta_{i 1}, \zeta_{i 2}, \ldots, \zeta_{i i-1}, 0, \zeta_{i i+1} \ldots \zeta_{i N}\right]$, where $i=1,2, \ldots, N$. Since $Z$ is an irreducible, row stochastic matrix, by the PerronFrobenius theorem [19, p.673], the eigenspace associated with the eigenvalue equal to 1 is one-dimensional, which implies that the equation $Z \gamma=\gamma$ has a unique solution given by $\gamma=(1, \ldots, 1)^{T}$, up to a scaling factor. This shows that $\gamma_{i}=\gamma_{j}$ for all $i, j$. Conversely, assume that $\left\{\alpha_{i j}\right\}_{i j}$ and $\left\{\psi_{i}\right\}_{i}$ satisfy constraint (16). Considering Lemma IV.2 (note, in particular, that $\gamma_{i}>0$ ), since $\gamma_{i}=\gamma_{j}$ for all $i, j$, then one immediately obtains that $\left\{\alpha_{i j}\right\}_{i j}$ and $\left\{\psi_{i}\right\}_{i}$ satisfy constraint (15). Hence, we can equivalently restate problem (14) as problem (10), which proves the claim.

Remarkably, problem (13) has the same form as the linear optimization problem in [22] used to find rebalancing policies within a fluidic model of an autonomous MOD system. In this respect, the analysis of our paper provides a theoretical foundation for the fluidic approximation performed in [22].

The importance of theorem IV.3 is twofold: it allows us to efficiently find an optimal open-loop, rebalancing promoting policy, and it enables the computation of quality of service metrics (namely, vehicle availability) for autonomous MOD systems as shown next.

\section{B. Computation of performance metrics}

By leveraging Theorem IV.3 one can readily compute performance metrics (i.e. vehicle availability) for an autonomous MOD system. First, we compute an optimal solution to the ORP using Theorem IV.3, which involves solving a linear optimization problem with $N^{2}$ variables. Next, we compute the relative throughputs $\pi$ 's using Lemma IV.1. Finally, we apply a well-known technique called mean value analysis (MVA) [23] in order to avoid the explicit computation of the normalization constant in equation (4), which is prohibitively expensive for large numbers of vehicles and stations. The MVA algorithm is an iterative algorithm to calculate the mean waiting times $W_{i}(n)$ and the mean queue lengths $L_{i}(n)$ at each node $i$ of a closed separable system of queues, where $n=1, \ldots, m$ is the numbers of vehicles over which the algorithm iterates. For the Jackson model in Section III-B, subject to the initial conditions $W_{i}(0)=L_{i}(0)=0$, the equations for MVA read as :

- $W_{i}(n)=\frac{1}{\mu_{i}(1)}=T_{\operatorname{Parent}(i) \operatorname{Child}(i)} \quad \forall i \in I$,

- $W_{i}(n)=\frac{1}{\mu_{i}}\left(1+L_{i}(n-1)\right)=\frac{1}{\tilde{\lambda}_{i}}\left(1+L_{i}(n-1)\right) \quad \forall i \in S$,

- $L_{i}(n)=\frac{n \pi_{i} W_{i}(n)}{\sum_{j \in \mathcal{N}} \pi_{j} W_{j}(n)} \quad \forall i \in \mathcal{N}$.

Finally, the throughput (or mean arrival rate of vehicles) to each station is given by Little's theorem [3, p.152]: $\Lambda_{i}(m)=$ $L_{i}(m) / W_{i}(m)$ for all $i \in S$. Combining equations (3), (2) and (4), one readily obtains the availability at each station as $A_{i}(m)=\Lambda_{i}(m) / \tilde{\lambda}_{i}$.

This procedure scales well to a large number of stations and vehicles, and is applied in Section $\nabla$ to real-world settings involving hundreds of stations and thousands of vehicles, to assess the potential performance of an autonomous MOD system in New York City.

The rebalancing promoting policy considered so far, while providing useful insights into the performance and operations of an autonomous MOD system, is ultimately an open-loop policy and hence of limited applicability. In the next section, we use insights gained from the ORP to formulate a closedloop rebalancing policy for the robotic vehicles that appears to perform well in practice.

\section{Real-time rebalancing policy}

In this section, we introduce a practical real-time rebalancing policy that can be implemented on real autonomous MOD systems. In reality, customers arriving at a station would wait in line rather than leave the system immediately (as in the loss 
model) if a vehicle is not available. In the mean time, information could be collected about the customer's destination and used in the rebalancing process. Let $v_{i}^{\text {own }}(t)$ be the number of vehicles "owned" by station $i$, that is, vehicles that are at station $i$, on their way to station $i$, or will be on their way to station $i$. We can write $v_{i}^{\text {own }}(t)=v_{i}(t)+\sum_{j} v_{j i}(t)+\sum_{j} \tilde{c}_{j i}(t)$, where $v_{i}(t)$ is the number of vehicles at station $i, v_{j i}(t)$ is the number of vehicles enroute from station $j$ to $i$, and $\tilde{c}_{j i}$ is the number of passengers at station $j$ that are about to board an available vehicle to station $i$. Note that $\sum_{i} \tilde{c}_{j i}(t) \leq v_{j}(t)$. Let $v_{i}^{e}(t):=v_{i}^{\text {own }}(t)-c_{i}(t)$ be the number of excess vehicles there will be at station $i$, where $c_{i}(t)$ is the number of customers at station $i$. The total number of excess vehicles is given by $\sum_{i} v_{i}^{e}(t)=\sum_{i}\left(v_{i}(t)+\sum_{j} v_{j i}(t)+\sum_{j} \tilde{c}_{j i}(t)-c_{i}(t)\right)=$ $m+\sum_{i} \min \left\{v_{i}(t), c_{i}(t)\right\}-\sum_{i} c_{i}(t)=m-\sum_{i} \max \left\{c_{i}(t)-\right.$ $\left.v_{i}(t), 0\right\}$. The second equality replaces $v_{i}(t)+\sum_{i} v_{j i}(t)$ with the total number of vehicles and asserts that in the current time step, either all of the customers or all the vehicles will leave the station. The last equality is obtained by considering both cases when $c_{i}(t) \geq v_{i}(t)$ and when $c_{i}(t)<v_{i}(t)$.

Through rebalancing, we may wish to distribute these excess vehicles evenly between all the stations, in which case each station will have no less than $v_{i}^{d}(t)$ vehicles, given by $v_{i}^{d}(t)=\left\lfloor\left(m-\sum_{i} \max \left\{c_{i}(t)-v_{i}(t), 0\right\}\right) / N\right\rfloor$. Accordingly, every $t_{\text {hor }}>0$ time periods, the number of vehicles to rebalance from station $i$ to $j$ num $_{i j}$, is computed by solving the linear integer optimization problem

$$
\begin{array}{ll}
\underset{\text { num }_{i j}}{\operatorname{minimize}} & \sum_{i, j} T_{i j} \operatorname{num}_{i j} \\
\text { subject to } & v_{i}^{e}(t)+\sum_{j \neq i}\left(\operatorname{num}_{j i}-\operatorname{num}_{i j}\right) \geq v_{i}^{d}(t) \quad \forall i \in S \\
& \operatorname{num}_{i j} \in \mathbb{N} \quad \forall i, j \in S, i \neq j
\end{array}
$$

This rebalancing policy takes all the current information known about the system and sets the rebalancing rates (in this case, the number of rebalancing vehicles) so that excess vehicles are distributed evenly to all the stations. This is in part inspired by the optimization problem in Theorem IV.3 It can be shown that the constraint matrix is totally unimodular, and the problem can be solved as a linear program, as the resulting solution will be necessarily integer-valued [22, Section 5]. The rebalancing policy presented here is closely related to the one presented in [22], the main difference being the inclusion of the current customers in line within the optimization process.

The real-time rebalancing policy will be used in Section $V$ to validate the vehicle availability performance criterion.

\section{Case Study: Autonomous MOD in Manhattan}

In this section we apply our availability analysis using the loss model to see how many robotic vehicles in an autonomous MOD system would be required to replace the current fleet of taxis in Manhattan while providing quality service at current customer demand levels. (We recently leveraged the theoretical results in this paper to perform a similar study for Singapore in [26].) In 2012, over 13,300 taxis in New York City made over 15 million trips a month or 500,000 trips a day, with around $85 \%$ of trips within Manhattan. Our study used taxi trip data collected on March 1, 2012 $2^{1}$ consisting of 439,950

\footnotetext{
${ }^{1}$ The data is courtesy of the New York City Taxi \& Limousine Commission.
}

trips within Manhattan. First, trip origins and destinations are clustered into $N=100$ stations throughout the city using $k$ means clustering. The resulting locations of the stations are such that a demand is on average less than $300 \mathrm{~m}$ from the nearest station, or approximately a 3-minute walk. The system parameters $\lambda_{i}, p_{i j}$, and $T_{i j}$ are estimated for each hour of the day using trip data between each pair of stations with Laplace smoothing. Some congestion effects are implicitly taken into account in the computation of $T_{i j}$, which uses the Manhattan distance and an average speed estimated from the data.

Vehicle availability is calculated for 3 cases - peak demand (29,485 demands/hour, 7-8pm), low demand (1,982 demands/hour, 4-5am), and average demand (16,930 demands/hour, 4-5pm). For each case, vehicle availability is calculated as a function of the fleet size using MVA techniques. The results are summarized in Figure 1(a).

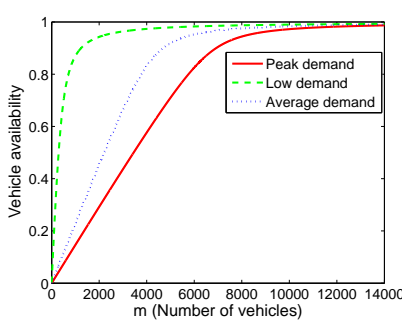

(a)

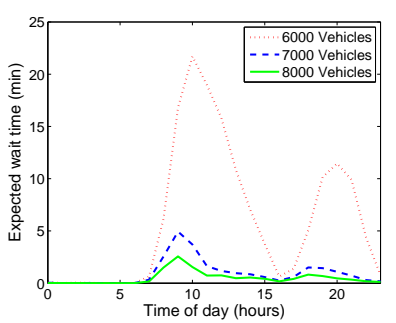

(b)
Fig. 1. 1(a) vehicle availability as a function of system size for 100 stations in Manhattan. Availability is calculated for peak demand (7-8pm), low demand (4-5am), and average demand (4-5pm). 1(b) Average customer wait times over the course of a day, for systems of different sizes.

For high vehicle availability (say, 95\%), we would need around 8,000 vehicles $(\sim 70 \%$ of the current fleet size operating in Manhattan, which, based on taxi trip data, we approximate as $85 \%$ of the total taxi fleet) at peak demand and 6,000 vehicles at average demand. This suggests that an autonomous MOD system with 8,000 vehicles would be able to meet $95 \%$ of the taxi demand in Manhattan, assuming 5\% of passengers are impatient and are lost when a vehicle is not immediately available. However, in a real system, passengers would wait in line for the next vehicle rather than leave the system, thus it is important to determine how vehicle availability relates to customer waiting times. We characterize the customer waiting times through simulation, using the realtime rebalancing policy described in Section IV-C. Figure 2 shows a snapshot of the simulation environment with 100 stations and 8,000 vehicles. Simulation are performed with discrete time steps of 2 seconds and a simulation time of 24 hours. The time-varying system parameters $\lambda_{i}, p_{i j}$, and average speed are piecewise constant, and change each hour based on values estimated from the taxi data. Travel times $T_{i j}$ are based on average speed and Manhattan distance between $i$ and $j$, and rebalancing is performed every 15 minutes. Three sets of simulations are performed for 6,000, 7,000, and 8,000 vehicles, and the resulting average waiting times are shown in Figure 1(b).

Figure 1(b) shows that for a 7,000 vehicle fleet, the peak averaged wait time is less than 5 minutes (9-10am) and for 8,000 vehicles, the average wait time is only 2.5 minutes. The simulation results show that high availability (90-95\%) does 


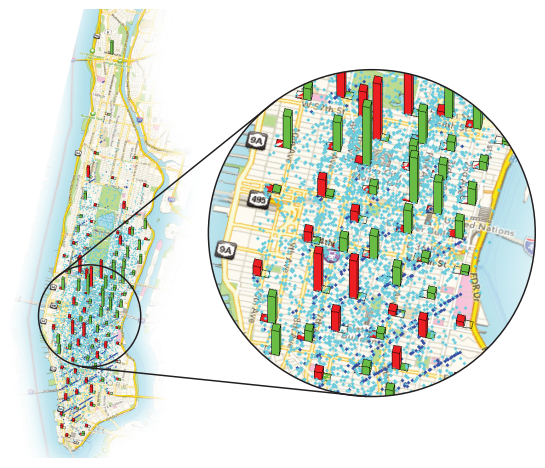

Fig. 2. Simulation environment with 100 stations in Manhattan. Red bars indicate waiting customers, green bars indicate available vehicles, cyan dots are vehicles travelling with passengers and blue dots are rebalancing vehicles.

indeed correspond to low customer waiting time and that an autonomous MOD system with 7,000 to 8,000 vehicles (50$60 \%$ of the size of the current taxi fleet) can provide adequate service with current taxi demand levels in Manhattan.

\section{Vi. A Mean Value Analysis Approach to the ANALYSIS OF CONGESTION EFFECTS}

The queueing model described in Section III does not consider congestion effects (roads are modeled as infinite server queues, so the travel time for each vehicle is independent of all other vehicles). However, if too many rebalancing vehicles travel on a route that is already congested, they can cause a traffic jam and decrease throughput in the entire system. Hence, in some scenarios, adding robotic vehicles to improve the quality of service might indeed have the opposite effect.

In this section, we propose an approach to study congestion effects that leverages our queueing-theoretical setup. The key idea is to change the infinite-server road queues to queues with a finite number of servers, where the number of servers on each road represents the capacity of that road. This road congestion model is similar to "vertical queueing" models that have been used in congestion analysis for stop-controlled intersections [18] and for traffic assignment [13]. In traditional traffic flow theory [17], the flow rate of traffic increases with the density of vehicles up to a critical value at which point the flow decreases, marking the beginning of a traffic jam. By letting the number of servers represent the critical density of the road, the queueing model becomes a good model for traffic flow up to the point of congestion.

Remarkably, the Jackson network model presented in Section III can be extended to the case where roads are modeled as finite-server queues; furthermore, the results presented in Section $[\Pi$ are equally valid. However, the travel times are no longer simply equal to the inverse of the service rates of the road queues, which significantly complicates the formulation of an analogue of problem ORP. While the issue of finding optimal rebalancing policies in the presence of congestion effects is left for future research, in this paper we show how given a rebalancing policy one can compute performance metrics such as vehicle availability (for example, one can study the effects of congestion on the performance of the rebalancing policies considered in Section IV].

In our approach, we first model the road network as an abstract queueing network with finite-server road queues, then we apply an extended version of the MVA algorithm for finiteserver queues, the details of which is presented in [23].

\section{A. Mapping physical roads into finite-server road queues}

The main difficulty in mapping the capacities of the road network into the number of servers of the queueing model (or "virtual" capacities, denoted by $m_{i j}$ ) is that trips from different origins and destinations may share the same physical road.

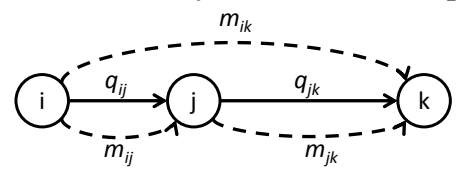

Fig. 3. A simple 3-station example showing the procedure of mapping physical roads into finite-server road queues.

As a simple example, consider the 3 -station network shown in Figure 3 Let $q_{i j}$ represent the maximum number of vehicles that can travel on the road between station $i$ and station $j$ without causing significant congestion. $m_{i j}$, the number of servers between $i$ and $j$, represents the number of vehicles that can travel between $i$ and $j$ before delays occur due to queueing. In the simple network, to go from station $i$ to station $k$, one must pass through station $j$. Hence, one has the following consistency constraints

$$
m_{i j}+m_{i k} \leq q_{i j}, \quad m_{j k}+m_{i k} \leq q_{j k} .
$$

To maximize the overall road usage, we can define a quadratic objective that seeks to minimize the difference between the real road capacities and the sum of the virtual road capacities:

$$
\min _{m_{i j}, m_{j k}, m_{i k}}\left(m_{i j}+m_{i k}-q_{i j}\right)^{2}+\left(m_{j k}+m_{i k}-q_{j k}\right)^{2}
$$

However, this optimization problem, along with the constraints (18), does not yield a unique solution because nothing is assumed about the relative usage rates of the road queues. If relative road usage is known, the $m_{i j}$ 's can be assigned proportional to the amount of traffic between each pair of stations that use the road. Let $\pi_{i j}$ be the relative throughput of the road queue between station $i$ and $j$, consistent with the earlier definition. Heuristically, the throughputs $\left\{\pi_{i j}\right\}_{i j}$ may be obtained from the arrival rates and travel patterns of passengers or from the analysis of a given rebalancing policy assuming no congestion (according to the procedure discussed in Section IV-B. For the simple example, one can write

$$
m_{i k} \leq \frac{q_{i j} \pi_{i k}}{\pi_{i k}+\pi_{i j}}, \quad m_{i k} \leq \frac{q_{j k} \pi_{i k}}{\pi_{i k}+\pi_{j k}} .
$$

Similar constraints can be written for $m_{i j}$ and $m_{j k}$ so that (18) is satisfied.

For a general road network, let $B_{i j}$ be the set of possible non-cyclic paths from station $i$ to $j$ (assuming no back tracking) and $C_{b_{i j}}$ be the set of road segments along path $b_{i j} \in B_{i j}$. The number of possible paths from $i$ to $j$ is given by $\left|B_{i j}\right|$. Let $a_{i j}^{c}$ denote the fraction of trips from $i$ to $j$ that go through road segment $c=$ \{origin, destination , where $c \in C_{b_{i j}}$. Denote by $q_{c}$ the capacity of road segment $c$. For trips going through multiple road segments, the virtual road capacity is determined by the segment with the lowest capacity. One can then consider as virtual road capacities:

$$
m_{i j}=\sum_{b \in B_{i j}} \min _{c \in C_{b_{i j}}}\left\{\frac{q_{c} a_{i j}^{c} \pi_{i j}}{\sum_{k, l, s, \mathrm{s.t} . c \in C_{b_{k l}}} a_{k l}^{c} \pi_{k l}}\right\} .
$$


In the next section we will apply this approach to study congestion effects for autonomous MOD systems on a very simple transportation network.

\section{B. Numerical study of congestion effects}

In this section we use a simple 9-station road network (shown in Figure 4) to illustrate the impact of rebalancing vehicles on congestion. The stations are placed on a square
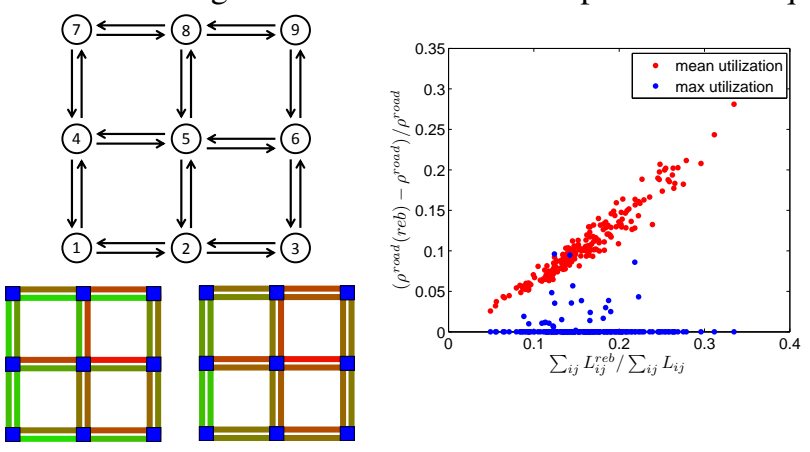

Fig. 4. Top left: Layout of the 9-station road network. Each road segmen has a capacity of 40 vehicles in each direction. Bottom left: The first picture shows the 9-station road network without rebalancing. The color on each road segment indicates the level of congestion, where green is no congestion, and red is heavy congestion. The second picture is the same road network with rebalancing vehicles. Right: The effects of rebalancing on congestion. The $\mathrm{x}$-axis is the ratio of rebalancing vehicles to passenger vehicles on the road The $\mathrm{y}$-axis is the fractional increase in road utilization due to rebalancing.

grid, and joined by 2-way road segments each of which is 0.5 $\mathrm{km}$ long. Each road consists of a single lane, with a critical density of 80 vehicles $/ \mathrm{km}$. 2 This means that the capacity of each road segment $c$ is $q_{c}=40$ vehicles. Each vehicle travels at $30 \mathrm{~km} / \mathrm{h}(8.33 \mathrm{~m} / \mathrm{s})$ in free flow, which means the travel time along each road segment is 1 minute in free flow.

To gain insight into the general system behavior, a variety of systems with different levels of imbalance must be studied. First, arrival rates and routing distributions are randomly generated and rebalancing rates are computed using (13). In steady state, the fraction of vehicles in each road queue $i j$ is given by $\pi_{i} \tilde{p}_{i j}$ (Lemma IV.1). If we assume $100 \%$ availability $\left(A_{i}=1\right)$, the expected rate of vehicles entering each road queue is given by $\Lambda_{i j}=\lambda_{i} p_{i j}$. Using Little's theorem, the expected number of vehicles on each road queue is given by $L_{i j}=\Lambda_{i j} T_{i j}$. The availability assumption can be justified by the fact that a real system would operate within the regime of high availability and that the number of vehicles on the road gets very close to $L_{i j}$ as availability increases. Similarly, the expected number of rebalancing vehicles on each road queue is given by $L_{i j}^{\mathrm{reb}}=\beta_{i j} T_{i j}$.

To map the queueing network onto the road network, we adopt a similar procedure as the one used to estimate $m_{i j}$ in Section VI-A Recall that $B_{i j}$ is the set of paths from station $i$ to station $j$. We adopt the routing strategy that uniformly distributes vehicles from $i$ to $j$ along each path $b_{i j} \in B_{i j}$. The number of vehicles that go through each road segment, $L_{c}^{\text {road }}$, is then the sum of the number of vehicles from each station to every other station that pass through the road segment, given by $L_{c}^{\text {road }}=\sum_{i, j \text {,s.t. } c \in C_{b_{i j}}} L_{i j} /\left|B_{i j}\right|$. Note that for stability, $L_{c}^{\text {road }}<q_{c}$. The road utilization is given by $\rho_{c}^{\text {road }}=L_{c}^{\text {road }} / q_{c}$.

\footnotetext{
${ }^{2}$ If each vehicle is $5 \mathrm{~m}$, this critical density represents a vehicle-to-vehicle separation of 1.5 car-lengths.
}

Figure 4 plots the vehicle and road utilization increases due to rebalancing for 500 randomly generated systems. The $\mathrm{x}$-axis shows the ratio of rebalancing vehicles to passenger vehicles on the road, which represents the inherent imbalance in the system. The red data points represent the increase in average road utilization due to rebalancing and the blue data points represent the utilization increase in the most congested road segment due to rebalancing. It is no surprise that the average road utilization rate is a linear function of the number of rebalancing vehicles. However, remarkably, the maximum congestion increases are much lower than the average, and are in most cases, zero. This means that while rebalancing generally increases the number of vehicles on the road, rebalancing vehicles mostly travel along less congested routes and rarely increase the maximum congestion in the system. This can be seen in Figure 4 bottom left, where rebalancing clearly increases the number of vehicles on many roads but not on the most congested road segment (from station 6 to station 5).

In a few rare cases, the maximum congestion in the system is increased up to $10 \%$. This may cause heavy congestion in systems where congestion is already prevalent (say, >90\%). In these cases, an intelligent routing strategy becomes crucial. While uniform routing along different paths helps distribute vehicles throughout the road network, a better routing strategy would actively route vehicles away from congested roads and perhaps even limit rebalancing when it may cause further delays. This is related to the simultaneous departure and routing problem [13], a class of dynamic traffic assignment (DTA) problems, and will be the subject of future work.

\section{CONCLUSIONS}

In this paper we presented and analyzed a queueingtheoretical model for autonomous MOD systems. We showed that an optimal open-loop policy can be readily found by solving a linear program. Based on this policy, we developed a closed-loop, real-time rebalancing policy that appears to be quite efficient, and we applied it to a case study of New York City. Finally, we showed that vehicle rebalancing can have a detrimental impact on traffic congestion in already-congested systems, but in most cases, rebalancing vehicles tend to travel along less congested roads.

This paper leaves numerous important extensions open for further research. First, it is of interest to develop rebalancing policies that can both route rebalancing vehicles along less congested roads and limit the number of rebalancing vehicles when the system is overly congested. Second, we plan to study different performance metrics (e.g., minimization of waiting times) and include a richer set of constraints (e.g., time windows to pick up the customers). Third, it is of interest to include in the model the provision of mass transit options (e.g., a metro) and develop optimal coordination algorithms for such an intermodal system. Fourth, we plan to extend the theoretical model to more realistic time-varying customer arrival rates. Fifth, we plan to consider additional case studies (e.g., from Asia and Europe) and study in more details the economic and societal benefits of robotic MOD systems. Finally, we plan to demonstrate the algorithms on real driverless vehicles providing MOD service in a gated community. 


\section{REFERENCES}

[1] M. Barth, J. Han, and M. Todd. Performance evaluation of a multi-station shared vehicle system. In Proceedings 2001 IEEE Intelligent Transportation Systems, pages 1218-1223. IEEE, 2001.

[2] G. Berbeglia, J. F. Cordeau, and G. Laporte. Dynamic pickup and delivery problems. European Journal of Operational Research, 202(1):8 - 15, 2010.

[3] D. P. Bertsekas, R. G. Gallager, and P. Humblet. Data networks, volume 2. Prentice-Hall International, 1992.

[4] F. Bullo, E. Frazzoli, M. Pavone, K. Savla, and S. L. Smith. Dynamic vehicle routing for robotic systems. Proceedings of the IEEE, 99(9):1482-1504, 2011.

[5] L. Burns, W. Jordan, and B. Scarborough. Transforming personal mobility. The Earth Institute, 2013.

[6] CAR2GO. CAR2GO Austin. Car Sharing 2.0: Great Idea for a Great City Technical report, 2011.

[7] M. Dell'Amico, E. Hadjicostantinou, M. Iori, and S. Novellani. The bike sharing rebalancing problem: Mathematical formulations and benchmark instances. Omega, 45(0):7 - 19, 2014.

[8] L. Di Gaspero, A. Rendl, and T. Urli. Constraintbased approaches for balancing bike sharing systems. In Principles and Practice of Constraint Programming, volume 8124 of Lecture Notes in Computer Science, pages 758-773. Springer Berlin Heidelberg, 2013.

[9] A. Fisher. Inside Google's Quest To Popularize SelfDriving Cars. Popular Science (Online Article), 2013.

[10] C. Fricker and N. Gast. Incentives and regulations in bike-sharing systems with stations of finite capacity. 2012. Available at http://arxiv.org/abs/1201.1178.

[11] D. K. George and C. H. Xia. Fleet-sizing and service availability for a vehicle rental system via closed queueing networks. European Journal of Operational Research, 211(1):198-207, 2011.

[12] GM. EN-Vs Impress Media at Consumer Electronics Show, 2011.

[13] H. Huang and W. H. K. Lam. Modeling and solving the dynamic user equilibrium route and departure time choice problem in network with queues. Transportation Research Part B: Methodological, 36(3):253-273, 2002.

[14] Induct. Navia - The 100\% Electric Automated Transport, 2013.

[15] R. C. Larson and A. R. Odoni. Urban operations research. Prentice-Hall, 1981.

[16] S. S. Lavenberg. Computer performance modeling handbook, volume 4. Elsevier, 1983.

[17] H. Lieu. Revised monograph on traffic flow theory. US Department of Transportation Federal Highway Administration, 2003.

[18] S. M. Madanat, M. J. Cassidy, and M. Wang. Probabilistic delay model at stop-controlled intersection. Journal of transportation engineering, 120(1):21-36, 1994.

[19] C. D. Meyer. Matrix Analysis and Applied Linear Algebra. SIAM, 2000.

[20] W. J. Mitchell, C. E. Borroni-Bird, and L. D. Burns. Reinventing the Automobile: Personal Urban Mobility for the 21st Century. The MIT Press, Cambridge, MA, 2010.
[21] M. Pavone, E. Frazzoli, and F. Bullo. Adaptive and distributed algorithms for vehicle routing in a stochastic and dynamic environment. IEEE Transactions on Automatic Control, 56(6):1259-1274, 2011.

[22] M. Pavone, S. L. Smith, E. Frazzoli, and D. Rus. Robotic load balancing for mobility-on-demand systems. The International Journal of Robotics Research, 31(7):839854, 2012.

[23] M. Reiser and S. S. Lavenberg. Mean-value analysis of closed multichain queuing networks. Journal of the ACM (JACM), 27(2):313-322, 1980.

[24] R. Serfozo. Introduction to stochastic networks, volume 44. Springer, 1999.

[25] S. L. Smith, M. Pavone, E. Schwager, E. Frazzoli, and D. Rus. Rebalancing the rebalancers: Optimally routing vehicles and drivers in mobility-on-demand systems. In American Control Conference (ACC), 2013, pages 23622367. IEEE, 2013.

[26] K. Spieser, K. Treleaven, R. Zhang, E. Frazzoli, D. Morton, and M. Pavone. Toward a systematic approach to the design and evaluation of automated mobility-ondemand systems: a case study in Singapore. Road Vehicle Automation, 2014.

[27] K. Treleaven, M. Pavone, and E. Frazzoli. Asymptotically optimal algorithms for pickup and delivery problems with application to large-scale transportation systems. IEEE Transactions on Automatic Control, 58 (9):2261-2276, 2013.

[28] UN. World Urbanization Prospects: The 2011 Revision Population Database Technical report, United Nations, 2011.

[29] A. Waserhole and V. Jost. Pricing in vehicle sharing systems: Optimization in queuing networks with product forms. 2013.

[30] R. Zhang and M. Pavone. Control of robotic mobilityon-demand systems: a queueing-theoretical perspective. 2014. Available at http://arxiv.org/abs/1404.4391. 